\title{
L'innovation en milieu hypernormalisé. Le cas des préposés aux bénéficiaires dans les organisations gériatriques au Québec
}

\section{The innovation in hyper-normalized environment. The case of the nursing assistants in the geriatric organizations in Quebec La innovación en medio hypernormalizado. El caso de los ayudantes sanitarios en las organizaciones geriátricas en Quebec}

François Aubry

Numéro 53, automne 2012

Sociologie et innovation

URI : https://id.erudit.org/iderudit/1023189ar

DOI : https://doi.org/10.7202/1023189ar

Aller au sommaire du numéro

Éditeur(s)

Athéna éditions

ISSN

0831-1048 (imprimé)

1923-5771 (numérique)

Découvrir la revue

Citer cet article

Aubry, F. (2012). L'innovation en milieu hypernormalisé. Le cas des préposés aux bénéficiaires dans les organisations gériatriques au Québec. Cahiers de recherche sociologique, (53), 11-32. https://doi.org/10.7202/1023189ar
Résumé de l'article

L’objectif général de cet article est de questionner les modalités d'appropriation par les préposés aux bénéficiaires de programmes innovants portant sur la bonification de leurs pratiques dans le contexte gériatrique québécois. À partir de l'exemple de la formation continue en emploi AGIR, qui promeut de nouvelles compétences en termes d'assistance aux résidents, et après avoir questionné trente préposés aux bénéficiaires à ce sujet, nous montrons que la diffusion d'innovations formatives ciblant les préposés individuellement n'occasionne pas nécessairement, selon ces derniers, une véritable amélioration des pratiques. Nous montrons que la raison de cet échec relatif tient à la non-prise en compte de la dimension collective de la pratique des préposés dans le cadre de la construction et de la diffusion de l'innovation. Nous postulons qu'un tel programme connaîtrait de meilleurs résultats si cette dimension était prise en compte dans la phase d'invention puis de diffusion de l'innovation. 


\section{L'innovation en milieu hypernormalisé. Le cas des préposés aux bénéficiaires dans les organisations gériatriques au Québec}

FRANÇOIS AUBRY

\section{Introduction}

L'objectif général de cet article est de questionner les modalités d'appropriation par les préposés aux bénéficiaires de programmes innovants portant sur la bonification de leurs pratiques dans le contexte gériatrique québécois. Trois sous-objectifs précisent notre idée. Il s'agit, d'abord, de montrer en quoi la plupart des programmes innovants portant sur le développement des pratiques de qualité ciblant les préposés aux bénéficiaires sont construits majoritairement sous la forme d'un processus individualisé de transfert des compétences; ensuite, nous voulons démontrer qu'une meilleure appropriation de ces programmes innovants serait facilitée s'ils étaient construits à partir de la dimension collective de la pratique des préposés. Enfin, nous postulons l'idée selon laquelle l'analyse sociologique du collectif de travail de préposés peut être une étape fondamentale de construction de nouveaux programmes innovants destinés aux intervenants des milieux de santé.

Une telle analyse nécessite de connaître étroitement la place des préposés dans le contexte organisationnel où l'innovation est implantée. D'une part, les préposés aux bénéficiaires sont des acteurs professionnels situés au bas de 
l'organisation gériatrique. Cela pose la question du rapport entre la position dominée de ce groupe professionnel et le mode d'appropriation des innovations prescrites: la diffusion des innovations peut-elle s'effectuer autrement que sous la forme de l'imposition de savoirs et de compétences à des acteurs professionnels singuliers? D'autre part, les centres d'hébergement et de soins de longue durée (CHSLD) peuvent être considérés comme des organisations particulières à un double niveau: celui de la gestion de l'organisation où se trament des prescriptions souvent incohérentes destinées à répondre aux enjeux en termes de charge de travail et qualité de pratiques ${ }^{1}$; puis celui du travail effectif où se retrouvent des normes de travail et des stratégies de régulation érigées et partagées par le collectif de préposés afin de parvenir à gérer ces enjeux contradictoires ${ }^{2}$. En ce sens, l'organisation gériatrique peut être définie comme un milieu hypernormalisé, voire déontique ${ }^{3}$ : en effet, l'atteinte des exigences managériales et relationnelles dépend étroitement de la capacité des préposés de composer avec ces normes informelles par l'usage de stratégies collectives.

Les préposés sont donc pris entre la domination hiérarchique qu'ils subissent et la forme d'autonomie et d'informalité qu'ils expriment par la création et l'usage de stratégies collectives de régulation. Cependant, l'exigence du respect des normes collectives informelles de travail peut entraver l'intégration des nouvelles pratiques issues des programmes novateurs de formation. Dit autrement, les stratégies collectives de régulation et les normes collectives peuvent empêcher l'appropriation de l'innovation prescrite en termes de bonification individuelle de la qualité de pratique, puisque cette dimension individualisée de l'innovation peut ne pas revêtir de signification pour le collectif de préposés, ni atteindre son principe d'efficacité et sa fonction protectrice pour le groupe. Nous voulons donc montrer comment la dimension collective de la pratique des préposés peut être prise en compte dans la conception puis l'implantation de programmes destinés aux préposés, si l'on veut que l'appropriation de ces innovations soit pleinement réussie. À cette fin, nous nous baserons sur les données tirées d'une étude réalisée en 2012 portant sur l'appropriation dans deux CHSLD au Québec

1. François Aubry, «Les rythmes contradictoires de l'aide-soignante. Conséquences sur la santé au travail de rythmes temporels contradictoires, en France et au Québec», Temporalités, n 16, 2012 [En ligne: http//temporalites.revues.org/2237].

2. Antoine Bonnemain, Conflit éthique et confits de temporalités: une approche ergonomique de la maltraitance en EHPAD, Mémoire de Master 2, Paris, CNAM, 2010, Séverine Chevalley, Étude visant à comprendre les dynamiques collectives en jeu dans la transmission des savoirs professionnels au sein d'équipes de préposés aux bénéficiaires, Mémoire de Master 1, Montréal, Institut de recherche en santé et sécurité du travail (IRSST) et Université de Lorraine, 2012.

3. Christophe Dejours, «Entre inégalités individuelles et coopération collective: la question de l'autorité», dans Marlène Jouan et Sandra Laugier (dir.) Comment penser l'autonomie? Entre compétences et dépendances, Paris, PUF, 2009, p. 291-313. 
d'une formation (AGIR) destinée à bonifier les pratiques des préposés aux bénéficiaires.

\section{Problématique : les préposés aux bénéficiaires dans les CHSLD au Québec}

Les préposés aux bénéficiaires sont les intervenants paramédicaux chargés de poser les actes nécessaires d'assistance et d'hygiène envers les résidants en CHSLD, dans l'objectif de répondre à leurs besoins fonctionnels quotidiens. La nomenclature de leurs tâches répond à la définition générique du Personal Support Worker de l'Organisation mondiale de la santé ${ }^{4}$. Cognet ${ }^{5}$ précise $^{-}$ que trois types de tâches leur sont assignés: il s'agit des soins et des services directs à la personne (hygiène, soins somatiques et soins psychosociologiques), des soins et services à l'environnement de la personne (entretien ménager) et des tâches administratives. Selon Grenier ${ }^{6}$, on comptait 35550 préposés en 2007 au Québec, dont la majorité sont des femmes (79\%). De ce chiffre, près de 1300 préposés se retrouvent en gériatrie, en CHSLD, soit 3,6\% de l'ensemble des membres du métier.

Les préposés aux bénéficiaires sont situés au bas de la hiérarchie organisationnelle du milieu gériatrique, sous la supervision directe des infirmières auxiliaires, responsables des actes de nursing. Les infirmières, quant à elles, sont responsables de l'évaluation de l'état de santé des résidants ainsi que de la bonne gestion et de l'efficacité du trinôme d'intervenants. Enfin, le chef d'unité de vie est responsable de l'efficacité du service gériatrique, de la relation avec les familles, de la mise en place des programmes de prévention et de qualité, etc. Globalement, les préposés doivent effectuer des tâches de faible technicité puisqu'ils agissent dans une organisation très hiérarchisée où l'aspect technique dépend de la possession d'un diplôme. Cognet et Fortin ${ }^{7}$ et Cognet et Raigneau ${ }^{8}$ ont d'ailleurs montré la corrélation existante entre le genre et la hiérarchie professionnelle dans les organisations de santé; ils décrivent en quoi les services de santé de type CHSLD peuvent être considérés comme des organisations de délégation genrée des tâches, où le travail de faible technicité, proche du travail relationnel, revient majoritairement aux femmes.

\footnotetext{
4. Organisation mondiale de la santé, Classifying Health Workers: Mapping Occupations to the International Standard Classification, Genève, WHO, 2010.

5. Marguerite Cognet, «Les femmes, les services et le don», Cahiers de recherche sociologique, n 37, 2002, p. 51-77.

6. Nancy Grenier, Portrait de la main-d'cuvre. Préposés aux bénéficiaires (Rapport Grenier), Québec, Ministère de la Santé et des Services sociaux du Québec, 2008.

7. Marguerite Cognet et Sylvie Fortin, «Le poids du genre et de l'ethnicité dans la division du travail en santé », Lien social et politique, $n^{\circ}$ 49, 2003, p. 155-172.

8. Marguerite Cognet et Laurent Raigneau, «Le genre et l'ethnicité: les critères voilés de la délégation des actes en santé», Les cahiers du Gres, vol. 3, n 1, 2002, p. 25-38.
} 
Les CHSLD, dans lesquels travaillent les préposés, ont aujourd'hui «pour mission d'offrir un milieu de vie substitut à des adultes en perte d'autonomie fonctionnelle ou psychosociale qui ne peuvent plus demeurer dans leur milieu de vie naturel, et ce, en dépit du soutien de leurs proches ${ }^{9} » ;$ si leur rôle n'a pas changé ces dernières années, l'alourdissement clinique de la population de résidants, du fait d'un resserrement important des critères d'accès favorisant davantage le maintien des personnes âgées en perte d'autonomie dans leurs milieux familiaux, est à souligner. Dans les années 1990, le virage ambulatoire a en effet conduit à une baisse du nombre de CHSLD - de 410 en 1993 à 160 en $2001^{10}$-, à une plus grande sélection de l'entrée des résidants en CHSLD et à une augmentation du nombre de résidants par préposés (ou ratio). En parallèle, on constate un important essor des ressources privées d'hébergement, où la qualité de la formation des préposés est encore plus incertaine que dans le réseau public.

Ainsi, les préposés aux bénéficiaires peuvent être considérés comme les membres dominés d'une organisation très hiérarchisée, sous la supervision et la surveillance directes de nombreux professionnels de la santé reconnus pour leur compétence médicale. Cette domination s'illustre particulièrement par les soupçons concernant la qualité de leurs pratiques: n'étant pas légalement obligés de détenir un diplôme pour obtenir un poste en CHSLD ${ }^{11}$, les préposés font fréquemment l'objet de critiques portant particulièrement sur la qualité de leurs pratiques. Jugés potentiellement incompétents, voire parfois maltraitants ${ }^{12}$, ils sont fréquemment reconnus comme les membres d'un groupe professionnel peu discipliné, manquant de compétences autant individuelles que collectives, et qui seraient les responsables, au moins en partie, de la mauvaise qualité des services administrée aux résidants: le manque de temps, de compassion ou d'intérêt porté à la personne hébergée trouverait sa cause directe dans les mauvaises pratiques des préposés.

9. Vincent Lemieux, Pierre Bergeron, Clermont Bégin et Gérard Bélanger, Le système de santé au Québec, Québec, Les Presses de l'Université Laval, 2003, p. 105.

10. Ibid.

11. François Aubry, Karine Godin et Yves Couturier, "Les préposées aux bénéficiaires face au processus d'implantation de l'approche Milieu de vie en soins de longue durée: critiques et perspectives futures», Vie et Vieillissement, vol. 9, n³, 2011, p. 36-42. Notons néanmoins que la possession du diplôme d'études professionnelles (DEP) en assistance à la personne en établissement de santé est actuellement considérée par les services de ressources humaines des CHSLD du Québec comme une règle officieuse... précisément pour prévenir les éventuels risques de maltraitance du personnel non ou pas suffisamment formé.

12. Marie-Anne Dujarier, «Comprendre l'inacceptable: le cas de la maltraitance en gériatrie», Revue internationale de psychosociologie, vol. 19, $\mathrm{n}^{\circ}$ 8, 2002, p. 111-124. 


\section{La formation AGIR: un programme innovant d'amélioration des pratiques}

Depuis une vingtaine d'années, le modèle conceptuel de l'approche Milien de vie a fait son apparition. Cette approche fait partie des nouvelles directives ministérielles promulguant une stratégie d'amélioration de la relation entre les intervenants et les résidants en CHSLD. Afin de transformer le milieu de soin en un milieu de vie, c'est-à-dire dans l'optique d'une bonification des pratiques des préposés en situation de travail et d'une revalorisation de l'image des CHSLD, on a évalué la qualité des pratiques des préposés en $2003^{13}$. Constatant qu'une amélioration était requise quant à l'implantation durable de la philosophie Milieu de vie $e^{14}$, le ministère a offert une formation - la formation AGIR $^{15}$ - à la quasi-totalité des préposés travaillant en CHSLD publics ou privés conventionnés ${ }^{16}$ entre 2009 et 2011 . Cette formation se compose de huit ateliers de travail relatifs à différents aspects de la pratique des préposés, et veut soutenir la mise en application des directives relatives à l'approche Milien de vie. Les administrations de chaque CHSLD ont disposé de la liberté de choisir parmi l'offre d'ateliers celui ou ceux répondant le mieux aux besoins organisationnels de leur centre. Le tableau, p. 16, résume la formation AGIR.

Dans le cadre de cette étude, l'atelier 1, AGIR pour maintenir et améliorer l'autonomie. Communication et relation aidante avec l'usager, fut choisi par les deux CHSLD participants. Cet atelier affirme le principe voulant que les besoins des résidants en matière d'autonomie priment sur les logiques organisationnelles et professionnelles de prestations de soins. Le développement des compétences est explicité sous la forme du rapport individualisé des préposés aux résidants; il est par exemple spécifié que, à la suite de la formation, les intervenants devront «adapter leurs façons de communiquer selon les besoins particuliers et les capacités de l'usager», ou encore "utiliser des techniques de communication axées sur l'établissement d'une relation aidante $^{17}$ ». L'importance donnée à la dimension individuelle dans ce type de formation n'est pas surprenante: dans le cadre d'une approche de formation

13. Ministère de la Santé et des Services sociaux (MSSS), Un milieu de vie de qualité pour les personnes hébergées en CHSLD. Orientations ministérielles, Québec, Direction des communications du ministère de la Santé et des Services Sociaux, 2003.

14. Ministère de la Santé et des Services sociaux (MSSS), Un milieu de vie de qualité pour les personnes hébergées en CHSLD. Visites d'appréciation de la qualité des services, Québec, Direction des communications du ministère de la Santé et des Services sociaux, 2004.

15. Ministère de la Santé et des Services sociaux (MSSS), Agir auprès de la personne âgée. Programme de la formation pour les préposés aux bénéficiaires travaillant auprès des personnes âgées en perte d'autonomie, Québec, Direction des communications du ministère de la Santé et des Services sociaux, 2008.

16. Les CHSLD privés conventionnés doivent répondre aux normes de qualité des organisations publiques.

17. Ministère de la Santé et des Services sociaux (MSSS), 2008, op. cit., p. 29. 
Tableau 1

La formation AGIR

\begin{tabular}{|c|c|c|c|}
\hline \multicolumn{4}{|c|}{ Le préposé aux bénéficiaires (PAB) face aux usagers } \\
\hline $\begin{array}{l}\text { 1. AGIR pour } \\
\text { maintenir et améliorer } \\
\text { l'autonomie }\end{array}$ & $\begin{array}{l}\text { 2. AGIR pour } \\
\text { protéger }\end{array}$ & $\begin{array}{l}\text { 3. AGIR pour } \\
\text { entourer }\end{array}$ & $\begin{array}{l}\text { 4. AGIR pour } \\
\text { préserver }\end{array}$ \\
\hline $\begin{array}{l}\text { Communication } \\
\text { et relation aidante } \\
\text { avec l'usager }\end{array}$ & $\begin{array}{c}\text { Troubles cognitifs, } \\
\text { troubles de } \\
\text { comportement } \\
\text { et situations } \\
\text { de crise }\end{array}$ & $\begin{array}{l}\text { Soins palliatifs } \\
\text { de fin de vie }\end{array}$ & $\begin{array}{c}\text { Besoins } \\
\text { physiologiques } \\
\text { dans des contextes } \\
\text { de soins de courte } \\
\text { durée } \\
\text { de longue durée }\end{array}$ \\
\hline \multicolumn{2}{|c|}{ Le PAB face à l'équipe } & $\begin{array}{l}\text { Le PAB face } \\
\text { aux proches }\end{array}$ & $\begin{array}{l}\text { Le PAB face } \\
\text { à lui-même }\end{array}$ \\
\hline $\begin{array}{l}\text { 5. AGIR pour } \\
\text { collaborer }\end{array}$ & $\begin{array}{l}\text { 6. AGIR pour } \\
\text { sécuriser }\end{array}$ & $\begin{array}{l}\text { 7. AGIR pour } \\
\text { accompagner }\end{array}$ & $\begin{array}{l}\text { 8. AGIR pour } \\
\text { prévenir }\end{array}$ \\
\hline $\begin{array}{l}\text { Rôle } \\
\text { et contribution } \\
\text { à l'équipe }\end{array}$ & $\begin{array}{l}\text { Observations } \\
\text { pertinentes } \\
\text { et situations } \\
\text { à risque }\end{array}$ & $\begin{array}{l}\text { Communication } \\
\text { et relation } \\
\text { avec les proches }\end{array}$ & $\begin{array}{l}\text { Respect des droits } \\
\text { et de la dignité } \\
\text { et prévention } \\
\text { de la négligence } \\
\text { et des abus }\end{array}$ \\
\hline
\end{tabular}

centrée sur les compétences ${ }^{18}$, l'acteur professionnel est considéré comme la source première de pratiques à bonifier par le biais d'interventions ${ }^{19}$.

Malencontreusement, comme nous le verrons plus loin, cette posture conduit à l'impossible prise en compte par les gestionnaires de la dimension collective du travail des préposés ${ }^{20}$. Même si certains ateliers démontrent un intérêt au développement de la communication de l'équipe de travail ou à la collaboration interprofessionnelle (Atelier 5: AGIR pour collaborer), cette dimension ne recouvre pas la dimension collective du travail des préposés. En fait, même la collaboration au travail, qui pourtant concerne la collectivisation de la pratique, est considérée avant tout comme une attitude intersubjective. À ce titre, un tel mode de diffusion d'innovation, de type indivi-

18. Arnaud Dupray, Christophe Guitton et Sylvie Monchatre, Réfléchir la compétence. Approches sociologiques, juridiques, économiques d'une pratique gestionnaire, Toulouse, Octarès, 2003.

19. Cédric Frétigné, «La formation individualisée en entreprise d'entraînement. Entre théorie et pratiques», Travail et formation en éducation, n 6, 2010 [En ligne : http://tfe.revues.org/1345].

20. François Aubry, Yves Couturier et Francis Etheridge, «Conceiving the skills development of nurse aides working in geriatrics as a collective process», Innovation-RICEC, vol. 4, n 1, 2013 [En ligne: http://ricec.info/images/stories/articlerevue/volume4_N1/IRICEC_Vol4_1_Aubry_al.pdf ]. 
dualiste, est-elle une voie à privilégier dans le cadre spécifique des préposés en CHSLD?

D'ailleurs, dans les écrits portant sur le processus de diffusion de l'innovation, la question du mode d'appropriation est majeure. Alter ${ }^{21}$ note que l'innovation se définit par son processus de construction et de diffusion se composant de trois temps: elle prend tout d'abord la forme d'une invention locale, préparée pour sa diffusion; l'invention se confronte aux acteurs de terrain lors d'une appropriation progressive; enfin, la stabilisation de la situation se caractérise par la définition progressive de nouvelles règles, c'està-dire par l'institutionnalisation de l'innovation. Dans ce triple processus, la seconde étape - celle de l'appropriation - semble nettement déterminante: la réussite d'une innovation réside dans sa faculté à donner du sens aux yeux des individus, et à entrer dans les usages sociaux adaptés à la situation de travail des professionnels.

L'application de l'innovation dans un contexte organisationnel spécifique peut être considérée comme une forme d'action collective dont le succès est conditionnel à la cohérence que les acteurs peuvent lui donner collectivement. Dans le cadre de la formation AGIR, l'appropriation de la formation par les préposés aux bénéficiaires doit pourtant se faire a priori individuellement. Cela revient à interroger les modalités d'appropriation d'une formation individualisée pour un collectif de travail extrêmement cohésif. La création de sens et la traduction en cohérence de l'innovation individualisée par les acteurs de terrain peuvent-elles être assurées, malgré la dimension collective du groupe de préposés?

C'est pour répondre à cette question que nous présentons les résultats d'une étude portant sur l'appropriation de la formation AGIR par les préposés. Notons que nous ne nous intéressons pas ici à la forme didactique de la formation, mais plutôt à l'adéquation de son contenu sur le plan des compétences promues vis-à-vis des pratiques mises en œuvre collectivement par les préposés. Cela peut nous permettre d'expliquer en quoi le processus de diffusion d'une innovation formelle ne peut faire l'économie d'une étude sociologique portant sur les pratiques effectives des acteurs censés s'approprier l'innovation.

\section{Méthodologie}

L'étude fut réalisée entre janvier et octobre 2012, dans un centre de santé et de services sociaux (CSSS) de la région de Montréal comptant deux

21. Norbert Alter, L'innovation ordinaire, Paris, PUF, 2005. 
CHSLD. L'objectif central fut d'identifier les conditions entravant ou facilitant la mise en œuvre par les préposés aux bénéficiaires de l'approche Milieu de vie à travers leur exposition à la formation AGIR. Nous avons opté pour une méthodologie qualitative, de type semi-inductif ${ }^{22}$, parce que, d'une part, nous souhaitions collecter les représentations ${ }^{23}$ des préposés sur ce sujet et que, d'autre part, peu d'écrits ont été publiés sur cette thématique. Nous avons réalisé 30 entrevues avec des préposés aux bénéficiaires (hors personnel d'agences privées) travaillant sur un quart de jour ( $7 \mathrm{~h}-15 \mathrm{~h})$, soit 15 entrevues dans deux services du CHSLD 1, et 15 entrevues dans deux services pour le CHSLD 2. Les préposés participants sont à «temps complet» $(\mathrm{n}=10)$, à «temps partiel» $(\mathrm{n}=10)$ et à «temps partiel occasionnel », recrutés depuis moins de 6 mois $(n=10)$. Nous avons choisi des services de soins gériatriques, en excluant néanmoins les services spécialisés pour des maladies ou déficiences spécifiques, telles que les unités spécialisées dans le soin aux résidants atteints de la maladie d'Alzheimer. L'entrevue, de type semi-dirigée, a été construite à partir de deux thématiques ${ }^{24}$, soit: 1) la représentation des acteurs de la formation AGIR et de son contenu en termes de compétences et recommandations, et 2) les contraintes ou les éléments favorisant l'application des compétences et recommandations contenues dans la formation. Nous illustrerons certaines analyses soutenues par des extraits des discours des préposés qui seront identifiés par leurs statuts et années d'expérience.

\section{Résultats: le désintérêt des préposés aux bénéficiaires pour la formation AGIR}

Quelle est la représentation des préposés aux bénéficiaires à l'égard de la formation AGIR offerte? Répond-elle à leurs besoins en termes de compétences, de bonification des pratiques et de développement de la qualité de leur intervention? L'opinion des préposés à ce propos est contrastée. Cependant, la totalité note qu'une telle formation est en principe intéressante et pertinente dans la perspective d'une bonification de leurs pratiques. Acquérir des compétences afin de mieux maintenir l'autonomie de la personne âgée est considéré comme un processus positif par l'ensemble des préposés.

On doit s'occuper de l'autonomie des résidents à $100 \%$, dans l'idée de faire du milieu de vie dans le centre d'hébergement. Alors ce qu'on apprend avec la

22. François Guillemette, «L'approche de la Grounded Theory pour innover?», Recherches qualitatives, vol. 26, n 1 , 2006, p. 32-50.

23. Jean Poupart et Groupe de recherche interdisciplinaire sur les méthodes qualitatives, La recherche qualitative. Enjeux épistémologiques et méthodologiques, Montréal, Gaëtan Morin, 1998.

24. Lorraine Savoie-Zajc, «L'entrevue semi-dirigée», dans Benoit Gauthier (dir.), Recherche en sciences sociales: de la problématique à la collecte des données, Québec, Presses de l'Université du Québec, 2009, p. 337-360. 
formation AGIR, c'est sûr que ça nous permet de voir des choses qui vont nous aider à faire ça. (Préposé aux bénéficiaires, temps partiel, 5 ans d'ancienneté)

Cette perception positive de la formation est collectivement partagée par l'ensemble des préposés. Néanmoins, ils notent que cette formation pose deux problèmes majeurs. Tout d'abord, elle ne répond pas aux besoins primordiaux des préposés en termes de contenu. Les compétences manquantes ou à développer ne seraient pas tant celles relatives au maintien de l'autonomie des résidants, comme celles développées dans l'atelier de la formation AGIR, mais plutôt celles permettant de répondre adéquatement aux pathologies dont souffrent les résidants. De fait, les préposés se sentent compétents à mettre en œuvre des pratiques paramédicales, mais souhaitent compléter leurs connaissances par des savoirs issus d'une formation sociosanitaire, pour mieux structurer leurs pratiques d'assistance.

Quand on regarde ce qu'on apprend avec la formation, c'est intéressant, c'est sûr que ça répond en partie aux problèmes de l'autonomie des résidants... mais dans le fond, c'est des choses qu'on sait déjà, enfin qu'on a déjà vu en formation durant le DEP et puis qu'on revoit ici... c'est intéressant, mais ça répond pas directement aux problèmes qu'on a tous les jours. (Préposé aux bénéficiaires, temps partiel occasionnel, 2 ans d'ancienneté)

Les soucis majeurs qu'on rencontre tous les jours, c'est plutôt: Comment répondre aux résidants qui sont atteints d'Alzheimer ou d'autres troubles... Ça c'est des problèmes nouveaux qu'on connaît depuis une dizaine d'années. On devrait avoir une formation davantage centrée sur le médical... (Préposé aux bénéficiaires, temps complet, 21 ans d'ancienneté)

Cette critique portant sur le contenu de la formation permet de comprendre en quoi les préposés aux bénéficiaires souhaiteraient que leur point de vue soit davantage pris en compte dans le cadre de la construction et de la diffusion de nouvelles formations. Selon eux, leur opinion a une importance décisive puisqu'ils connaissent tout particulièrement les problématiques soulevées dans l'assistance aux résidants. En parallèle, ils critiquent le fait que des formations leur soient imposées par des intervenants extérieurs à leur pratique, qui a priori ne connaissent pas adéquatement les enjeux de la relation de proximité entre préposés et résidants.

On nous impose des formations, comme AGIR, on sous-entend un peu que les préposés ne savent pas travailler correctement... On ne prend jamais ou rarement l'avis des préposés dans la construction de ce type de formation ou de programme, alors qu'on est les yeux et les oreilles des résidants, on sait quelles compétences manquent ou quelles qualités il faudrait développer. (Préposé aux bénéficiaires, temps partiel occasionnel, 2 ans d'ancienneté)

Les préposés critiquent donc le fait qu'on les perçoive comme des intervenants qu'il faudrait instruire sans cesse afin qu'ils puissent réaliser un travail 
individuel de qualité. La suspicion de maltraitance est ressentie quotidiennement par les préposés qui critiquent cette perception négative dont ils font l'objet: ils émettent des critiques sur les jugements provenant des médias et, plus globalement, du monde extérieur à l'organisation.

Dans notre métier, on sait bien qu'on est mal vu... qu'on est dans le sale tous les jours, puis qu'on n'est pas bon... dès qu'il y un problème avec un résidant, c'est de la faute des préposés... on fait globalement du bon travail, mais au moindre problème, même si le préposé n'est pas responsable, c'est la meilleure cible... (Préposé aux bénéficiaires, temps partiel, 5 ans d'ancienneté)

Mais la critique la plus importante aux yeux des proposés a pour objet la hiérarchie de type hospitalier qui caractérise l'organisation du travail en CHSLD. Selon les préposés rencontrés, les responsables des formations qu'on leur propose sont déconnectés de l'organisation réelle du travail, du «plancher»: ils ne comprennent pas les enjeux réels auxquels l'ensemble des préposés est confronté. Les chefs d'unité de vie sont particulièrement visés par la critique, notamment lors de controverses portant sur la relation entre préposés et aidants familiaux, ou sur une pratique d'assistance jugée maladroite, voire dangereuse ou maltraitante.

La chef d'unité vient souvent nous achaler sur quelque chose qu'on a pas fait, ou qu'on a fait mal, ou par rapport à une famille qui s'est plainte. Comme il y a pas longtemps, je me suis fait critiquer sur le fait qu'une famille s'est plainte, mais le résidant est très demandant [...]. Les chefs peuvent venir faire des remarques, mais je pense qu'ils ne connaissent pas les résidants comme nous autres, et qu'ils ne connaissent pas les problèmes que nous on subit. (Préposé aux bénéficiaires, temps complet, 21 ans d'ancienneté)

Le discours des préposés montre qu'une séparation peut être identifiée entre, d'une part, les acteurs professionnels paramédicaux considérés proches du terrain, soit les préposés aux bénéficiaires, les infirmières auxiliaires et les infirmières et, d'autre part, les autres intervenants médicaux ou gestionnaires plus éloignés du terrain, c'est-à-dire les chefs d'unité de vie, les conseillers à la qualité et, de manière plus générale, la direction des centres d'hébergement. Les avis, critiques et conseils provenant de ces derniers sur le travail des préposés sont perçus plutôt négativement par les préposés, du fait qu'ils ne connaîtraient pas suffisamment la réalité et la complexité de leurs pratiques.

Les directions d'hébergement, ceux qui sont dans les bureaux, ils ne savent pas c'est quoi le travail de préposé... ils viennent jamais voir, par contre ils nous demandent de faire de meilleures pratiques, de mettre en place des approches comme Milieu de vie, de faire de plus en plus, de mieux en mieux, mais avec moins de moyens, moins de matériel... (Préposé aux bénéficiaires, temps complet, 18 ans d'ancienneté) 
Les préposés notent que la méconnaissance des gestionnaires concernant la complexité de leurs pratiques se résume à deux objets précis, soit les résidants et la charge de travail. D'une part, les préposés déplorent le fait que les membres de la direction ne connaissent pas suffisamment les besoins des résidants ainsi que les compétences qu'ils développent effectivement pour les assister. Les préposés se présentent plutôt comme les intervenants détenant des savoir-faire profanes ou domestiques invisibles à leurs supérieurs hiérarchiques, et que nul autre intervenant ne possède.

Les résidants, il faut être à côté d'eux au quotidien. C'est nous qui savons s'il a besoin de quelque chose ou pas, qui savons comment s'en occuper. Quand je vois la chef d'unité de vie arriver, et me dire: «Oh, une famille vient se plaindre que leur mère se sent mal aidée », et qu'elle nous critique et tout, je m'excuse, j'aimerais qu'elle prenne ma place, juste une journée, pour voir que ce que disent les familles et ce qu'on vit nous, ça a rien à voir. (Préposé aux bénéficiaires, temps partiel, 5 ans d'ancienneté)

D'autre part, les critiques des préposés envers leurs supérieurs portent également largement sur la question de la reconnaissance de la charge de travail et des conditions de travail. En effet, ils notent que l'écart ressenti entre la qualité des pratiques réclamée par les directions d'hébergement et la qualité effective ou jugée par les acteurs est fortement conditionnée par la charge de travail non identifiée par les membres de la direction. Les directions d'hébergement seraient, selon les préposés, directement responsables des mauvaises pratiques éventuelles envers les résidants en centre d'hébergement.

En effet, d'un côté, ils ne donneraient pas de moyens supplémentaires aux préposés - en termes de main-d'œuvre notamment - pour assurer une meilleure qualité des pratiques.

Si on est un préposé de plus le matin, notamment sur le quart de jour $7 \mathrm{~h}-15 \mathrm{~h}$, là on pourra faire du bon travail. Mais là, on ne peut pas... parce qu'on n'est pas assez nombreux pour pouvoir prendre le temps de faire des soins de qualité [...]. Et c'est des choses qu'on dit souvent à la direction, de rajouter du personnel, mais ils veulent rien savoir... pas de budget, soi-disant. (Préposé aux bénéficiaires, temps partiel, 4 ans d'ancienneté)

De l'autre côté, ces mêmes membres de la direction d'hébergement ne prendraient pas suffisamment en compte la charge de travail lorsqu'ils doivent juger une controverse avec une famille ou dans le cadre de problèmes pratiques ou organisationnels relatifs au travail des préposés. Selon ces derniers, il faudrait idéalement que les problématiques relatives à la qualité des pratiques, c'est-à-dire les controverses, les critiques, etc. portant sur la qualité de l'assistance avec le résidant, puissent être intimement mises en relation avec les enjeux en termes de charge de travail. 
On nous critique souvent sur la qualité de ce qu'on fait, si on s'occupe bien des madames, s'il y a des problèmes, c'est toujours nous qui sommes pris à part. Mais on ne regarde jamais tout le travail qu'on a à faire... c'est toujours des critiques sommaires mais personne ne regarde vraiment qu'on a 5, 6 voire 7 résidants parfois le matin, puis qu'on doit faire toutes les toilettes, et les repas... Ce n'est pas seulement une question de volonté, on manque de moyens humains, donc du personnel, puis des nouveaux équipements parfois. (Préposé aux bénéficiaires, temps complet, 18 ans d'ancienneté)

Ainsi, on peut comprendre que les préposés critiquent les modalités de diffusion d'innovation en termes de formation, telle que la formation AGIR: ce type de formation ne répondrait pas aux besoins véritables des préposés, concentrés plutôt autour de la réduction de la charge de travail. Cela ne veut pas dire que, pour les préposés, les compétences transmises ne sont pas intéressantes ou déconnectées de la réalité organisationnelle; mais celles-ci devraient plutôt être développées avec une prise en compte intime de la lourdeur de la tâche et des stratégies collectives compensatrices. Dit autrement, les préposés notent qu'ils ne peuvent pas améliorer la qualité de leur pratique par le seul biais d'une formation si la quantité de travail en termes de charge de travail ou d'intensification des rythmes de travail n'est pas considérée, dans le cadre de la formation, comme un enjeu majeur.

La formation aurait alors peut-être un autre destinataire que les préposés eux-mêmes, soit l'opinion publique en quête de réassurance à l'égard de la qualité des services en CHSLD.

La formation AGIR, c'est intéressant, mais ça répond pas directement à nos besoins... je veux dire: "Oui de nouvelles compétences pour mieux maintenir l'autonomie de la personne», OK, mais ça ne prend pas en compte la charge de travail qu'on a... et ça, je dirais que c'est pour presque toutes les formations pareils: c'est des bonnes idées, mais faudrait mieux prendre en compte ce qui nous bloque, et ça c'est la charge de travail. (Préposé aux bénéficiaires, temps partiel, 5 ans d'ancienneté)

Dans ce contexte, l'appropriation de l'innovation semble complexe, puisqu'elle ne répond pas à la culture professionnelle des préposés basée principalement sur la maîtrise des enjeux quantitatifs. En fait, une telle imposition de formation conduit les préposés à ressentir fortement leur position de dominés au sein de l'organisation: ils se voient imposer des formations affirmant tacitement leur incompétence, sans que leur point de vue soit pris en compte.

La suite de notre analyse montre que les préposés savent réguler leur pratique, jouer avec les règles prescrites et imposer des normes collectives pour parvenir à respecter les enjeux contradictoires de l'organisation, entre 
une lourde charge de travail imposée et de nouvelles exigences qualitatives en termes d'assistance envers les résidants.

\section{L'usage réitéré des stratégies collectives de régulation et hypernormalisation}

Nos résultats prouvent que les préposés sont méfiants vis-à-vis des membres de la hiérarchie organisationnelle, mais qu'ils font confiance aux acteurs près du "plancher», constituant ainsi un véritable collectif de travail, au sein d'une instance de partage de normes et de compétences collectives. Précisément, la culture organisationnelle des préposés se définit en grande partie par le travail de recréation collective de la tâche prescrite. Dit autrement, afin de répondre conjointement aux exigences quantitatives et qualitatives imposées par les directions d'hébergement, les préposés font preuve d'autonomie collective pour créer des moyens d'y arriver, notamment des stratégies de régulation des objectifs perçus comme contradictoires.

On veut faire du travail de qualité, mais on n'a pas forcément les moyens de faire tout comme on voudrait... Aider une personne qui veut aller à la toilette, c'est bien, mais ca prend du temps, et on en a 7 à s'occuper! On ne peut pas les assister tous comme ca. Alors on est toujours pris entre le fait de faire bien, mais le manque de temps pour le faire pour tout le monde... (Préposé aux bénéficiaires, temps complet, 15 ans d'ancienneté)

Répondre à ces exigences contradictoires est une difficulté ressentie par tous. En effet, chacun des préposés interrogés nous a fait part de cette représentation de la tâche. La conciliation d'objectifs contradictoires n'est donc pas l'apanage d'un préposé incompétent, mais une compétence collective invisible devant un problème organisationnel majeur.

On est tous pris avec le fait qu'on doit faire un travail d'assistance de bonne qualité... je veux dire que toute la direction, les chefs d'unité de vie jugent ce qu'on fait avec les résidants, mais en même temps on doit composer avec le fait qu'on en a plusieurs à faire durant le quart de jour, et pareil pour ceux du quart de soir. Et ça, cette pression-là, tout le monde la ressent. (Préposé aux bénéficiaires, temps partiel, 5 ans d'ancienneté)

La principale critique portée par les préposés aux bénéficiaires sur ces doubles exigences tient à la contradiction des rythmes de travail qu'elles occasionnent. Répondre à la forte charge de travail nécessite d'accélérer collectivement le rythme de travail, alors que les principes de qualité des pratiques et de maintien de l'autonomie des résidants, comme les prescrit la formation AGIR, réclament un rythme de travail individuel lent. Plus généralement, le temps est un enjeu fondamental des pratiques. Ils disent effectuer un véritable travail de régulation des temporalités; par exemple, ils notent qu'ils 
doivent limiter au maximum les phases de lenteur - c'est-à-dire les phases de proximité avec les résidants -, afin de terminer leur journée de travail «dans les temps». Concrètement, les préposés utilisent des stratégies de régulation des rythmes de travail basées sur leur connaissance collective des résidants et des pratiques d'assistance. Par exemple, l'un d'entre eux raconte comment, durant les toilettes matinales, et dans l'objectif d'assurer conjointement la qualité et la charge de la tâche, l'ensemble des préposés doit parvenir à gérer le temps nécessaire pour discuter avec le résidant tout en le lavant.

Le matin, c'est go, go, go... tu n'as pas le temps de rester avec la madame pour discuter avec. Tu discutes mais tu fais tes affaires en même temps. Donc, tu ouvres les rideaux, tu la lèves, tu lui fais sa toilette, tout ça en 15 minutes. Donc, ce n'est pas idéal, mais tu arrives à faire un peu de relationnel avec elle, puis après tu passes à une autre. (Préposé aux bénéficiaires, temps partiel, 5 ans d'ancienneté)

Cette pratique individuelle découle d'un ajustement collectif produisant une norme commune d'efficience du travail. Ces stratégies de régulation, nombreuses, ont donc pour fonction de réguler collectivement les rythmes de travail des préposés, c'est-à-dire de limiter les phases de ralentissement, tout en respectant autant que faire se peut, d'un point de vue collectif, les exigences qualitatives, notamment du point de vue du maintien de l'autonomie. Le non-respect de ces normes communes produirait une preuve d'incompétence chez tous les préposés n'arrivant pas à suivre cette cadence, par exemple chez un nouveau préposé.

Si des stratégies ont été répertoriées dans tous les services étudiées, leur nature diffère selon l'organisation. Elles peuvent être identifiées en deux catégories, dépendamment de leur caractère moralement acceptable au regard des principes de qualité d'assistance. Certaines, comme celle décrite précédemment, ne présentent pas d'enjeux moraux fondamentaux. Par contre, la stratégie de nourrir deux résidants en même temps peut poser certainement quelques problèmes de qualité de l'assistance (ne pas se tromper dans l'administration des repas, par exemple), mais peut être assurée publiquement, sans risque de désapprobation provenant des pairs ou de la hiérarchie, en raison de la norme collective affirmant la nécessité de maintenir la cadence. Selon les préposés, même les membres de leur hiérarchie directe (infirmière auxiliaire et infirmière, voire chef d'unité de vie) connaissent l'existence de ces stratégies, et n'en critiquent pas leur usage.

Pour aller plus vite, c'est quelque chose qu'on fait tous dans le service: on met deux résidants en face de nous, et on donne à manger alternativement à l'un et à l'autre. Ca permet d'aller plus vite, parce qu'ils sont très lents pour la plupart... et la direction d'hébergement est bien correcte avec ça. Les chefs d'unité 
le savent, les infirmières le savent... elles savent que ca permet d'aller plus vite. (Préposé aux bénéficiaires, temps complet, 18 ans d'ancienneté)

Mais d'autres stratégies prennent une dimension plus clandestine, tout en conservant leur dimension collective. Par exemple, les préposés doivent refuser de répondre à certaines demandes de résidents réclamant beaucoup de temps pour prioriser certaines tâches essentielles.

On a des résidants atteints d'incontinence, l'idée c'est qu'on devrait faire en sorte de développer leur autonomie, donc de les aider à aller à la toilette... Mais on ne peut pas le faire tout le temps. On doit plutôt prioriser, une fois durant le quart de matin. Alors ce qu'on fait, c'est qu'on répond aux cloches à environ $11 \mathrm{~h}$, c'est un moment plus calme, on a fini les toilettes et les déjeuners normalement. Mais on ne répond pas aux cloches de ces gens-là avant 11 h. (Préposé aux bénéficiaires, temps partiel, 3 ans d'ancienneté)

Cette catégorisation collective est une stratégie directe pour identifier les pratiques de travail chronophages, et en minimiser les effets sur certains résidants, mais pas sur tous. Mais, puisque cette norme tacite entre en contradiction avec les principes de qualité des pratiques et de maintien de l'autonomie, elle tend à s'effectuer plutôt clandestinement. De tels arrangements collectifs ne sont pas vécus positivement par les préposés, qui préféreraient donner une assistance de qualité grâce à davantage de temps.

On nous dit avec AGIR, qu'il faut prendre notre temps pour la personne, qu'on doit développer son autonomie, alors c'est sur que ca devient vite des pratiques qui prennent du temps [...] et quand je regarde notre travail, surtout durant le quart de jour, on ne peut pas prendre ce temps-là... On est sans cesse en train d'essayer de rattraper du temps; j'ai l'impression, certains matins, qu'on est déjà en retard avant de commencer. Alors ce n'est pas des bonnes conditions pour mettre en œuvre des pratiques soi-disant de maintien de l'autonomie, on n'a pas le temps pour ca. (Préposé aux bénéficiaires, temps partiel occasionnel, 3 ans d'ancienneté)

De fait, les pratiques prescrites aux préposés en termes de qualité ne peuvent pas leur convenir totalement, car elles s'éloignent trop de la réalité organisationnelle qu'ils subissent, c'est-à-dire des arrangements qu'ils doivent réaliser quotidiennement, publiquement ou clandestinement, pour respecter ces enjeux. On comprend pourquoi le contenu de la formation AGIR, en tant que pratiques idéales, peut rentrer en conflit avec la réalité du terrain, en délégitimant ces normes pragmatiques, et en les connotant comme étant des pratiques moralement (et donc individuellement) inappropriées. 


\section{Discussion. Le collectif de travail: une dimension à prendre en compte dans la création et la diffusion des innovations à destination des préposés aux bénéficiaires}

Les résultats de cette étude montrent tout l'intérêt d'étudier la dimension collective des pratiques de travail de préposés aux bénéficiaires comme une condition nécessaire à la création et la diffusion des innovations. La sociologie du travail offre les outils conceptuels et méthodologiques pour ce faire. Le collectif peut être ici défini comme une instance majeure qui conditionne les pratiques mises en œuvre en CHSLD. En somme, les pratiques effectuées en CHSLD par les préposés ne correspondent jamais totalement à celles diffusées par les formations et imposées par la hiérarchie. Au contraire, elles sont recréées, reconstruites par le collectif, afin de concilier des exigences organisationnelles qui, comme on a pu le voir, présentent des faces contradictoires.

Nos résultats remettent en cause les principes de diffusion des formations comme conditions favorables à l'implantation réussie d'innovations en CHSLD, comme la formation AGIR, basée sur le principe d'un transfert individualisé des compétences. En effet, les enjeux organisationnels auxquels les préposés tentent de répondre ne trouvent pas de solutions directes dans les compétences issues de la formation AGIR, mais plutôt dans les compétences collectives qu'ils mettent en ouvre. En ce sens, le désintérêt des préposés pour le contenu de la formation AGIR montre à quel point le transfert individualisé des compétences ne trouve pas de signification précise pour eux: le processus d'appropriation ne peut pas se faire, parce que les compétences imposées par cette formation ne correspondent pas aux compétences effectivement requises pour répondre à la réalité organisationnelle.

Le collectif doit alors être perçu comme une dimension incontournable de la recréation de la tâche, par l'effet notamment de la régulation des rythmes de travail. Le collectif détient trois fonctions majeures. Sa première fonction est d'abord d'être le moteur d'une recréation de la tâche prescrite en fonction des conditions réelles d'exécution de la tâche, une «recréation de l'organisation du travail par le travail d'organisation du collectif », comme le note $\mathrm{Clot}^{25}$. En ce sens, les stratégies de régulation peuvent être considérées comme des formes d'inventions collectives qui permettent de réduire les

25. Yves Clot, «La fonction psychologique du collectif», dans Benchekroun Tahar Hakim et Weill-Fassina Annie (dir.), Le travail collectif en ergonomie. Perspectives actuelles, Toulouse, Octarès, 2000, p. 273-274. 
tensions issues d'exigences contraignantes ou contradictoires, comme l'ont montré Caroly et $\mathrm{Clot}^{26}$ ou encore Bonnemain ${ }^{27}$.

Sa seconde fonction fait référence au partage et à la transmission de ces stratégies entre recrues et membres expérimentés. Afin que chacun puisse utiliser de telles stratégies, elles doivent être transmises rapidement, durant leur premier temps de pratique, ce qui n'est pas sans occasionner des difficultés, voire du désenchantement pour les recrues qui doivent faire face aux contraintes présentées plus haut ${ }^{28}$.

Enfin, sa troisième fonction, essentielle, tient à la dimension normative, voire déontique ${ }^{29}$ du collectif. Par là, nous voulons dire que le collectif canalise les individualités, les forme, pour que le groupe de préposés produise des pratiques relativement homogènes. Les conduites différentes, les transgressions, font l'objet de jugements critiques de la part des autres membres du collectif.

Nous pensons que, dans le cadre du processus de construction et de diffusion des innovations formatives, ce dynamisme du collectif devrait être pris en compte. Plus exactement, les innovations formatives devraient être conçues en rapport avec ce dynamisme collectif, afin de ne pas tomber en contradiction avec les pratiques mises en œuvre quotidiennement. Une telle proposition va à l'encontre de certains programmes actuels portant sur la formation des préposés aux bénéficiaires. La difficulté majeure provient de l'incapacité des gestionnaires du changement à considérer que les préposés peuvent être eux-mêmes les propres acteurs collectifs du changement organisationnel. L'idée selon laquelle ces acteurs professionnels dominés dans l'organisation gériatrique doivent acquérir des compétences qu'ils ne possèdent pas, faute de formation, de compétences, voire de savoir-vivre (puisque nous parlons de compétences «domestiques $\left.{ }^{30} »\right)$ empêche de considérer que le collectif peut être un levier efficace permettant la création et la diffusion des innovations.

Pour ce faire, l'acte premier de tout projet innovant consiste à comprendre la pratique réelle pour en faire remonter les savoirs utiles à la conception d'une formation véritablement innovante. Cela ne veut pas dire qu'il est impossible de soutenir le changement de pratique. Cependant, tout projet de transformation doit prendre appui sur une intelligence profonde des pos-

26. Sandrine Caroly et Yves Clot, «Du travail collectif au collectif de travail: Développer des stratégies d'expérience. Autour de l'expérience et de sa validation», Formation-Emploi, n 88, 2004, p. 43-55.

27. A. Bonnemain, op. cit.

28. François Aubry, «Transmettre un genre professionnel, l'exemple des nouvelles recrues aides-soignantes: une comparaison France - Québec », Formation-Emploi, 119, 2012, p. 47-63.

29. C. Dejours, op. cit.

30. M. Cognet, op. cit. 


\section{Figure 1}

Le rôle du collectif de préposés dans la gestion des prescriptions organisationnelles

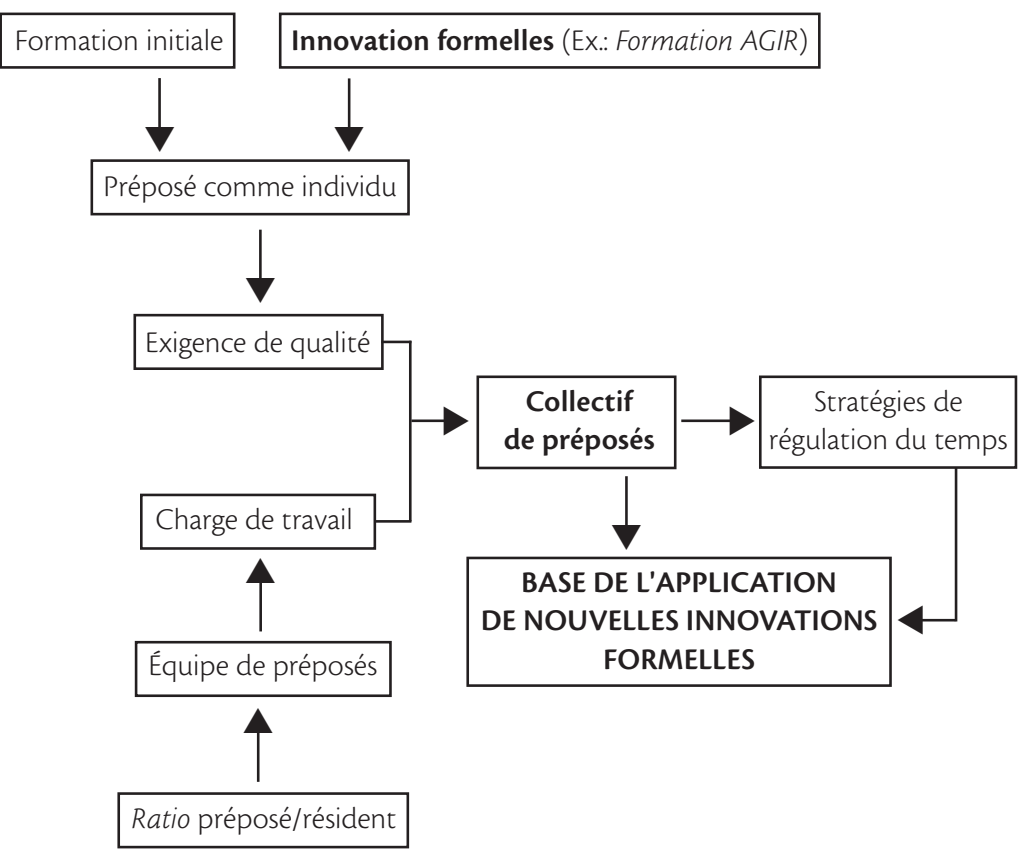

sibles de la situation d'intervention, ce à propos de quoi la didactique professionnelle nous invite à réfléchir ${ }^{31}$. La figure ci-dessus résume ce point de vue.

Construire un processus de création et de diffusion de l'innovation formative à partir des connaissances scientifiques portant sur le travail de régulation des préposés peut être une étape déterminante permettant l'appropriation réussie de l'innovation, puisque celle-ci prendra en compte la culture organisationnelle des préposés. Mais un tel projet d'utilisation du collectif nécessite une base importante, soit une connaissance approfondie des stratégies de régulation et des pratiques informelles des préposés (ou plus globalement, de la culture organisationnelle) ainsi qu'un modèle d'utilisation du collectif.

31. Patrick Mayen, «Passer du principe d'alternance à l'usage de l'expérience en situation de travail comme moyen de formation et de professionnalisation », dans France Merhan, Christophe Ronveaux et Sabine Vanhulle (dir.), Alternances en formation, Bruxelles, De Boeck, 2007, p. 83-100. 


\section{Une forme avancée d'étude et d'usage du collectif: empowerment en santé au travail}

Nos données montrent tout l'intérêt de prendre en compte la dimension collective dans la production de l'innovation pour que l'appropriation se révèle réussie. La puissance référentielle du collectif que nous avons mise en évidence fournit des pistes d'action pour soutenir le développement professionnel des préposés aux bénéficiaires. Les organisations gériatriques de type CHSLD ont une culture de gestion top-down, où la participation du personnel soignant est rare $^{32}$. Les préposés sont fréquemment oubliés dans le cadre d'efforts d'amélioration de la qualité, et ce, même s'ils passent le plus de temps auprès des résidants $^{33}$. Il existe une homologie de la souffrance entre celle de résidants et celle des travailleurs au bas de la hiérarchie organisationnelle ${ }^{34}$.

Il serait pourtant possible de renverser la proposition et de concevoir le collectif de préposés comme une force positive d'adaptation aux contraintes, par laquelle des stratégies de promotion ou de formation seraient possibles, mais aussi une stratégie d'habilitation et d'autonomisation de ce groupe professionnel si crucial. Une meilleure prise en compte de ce collectif pourrait permettre l'émergence d'une gestion plus participative, principe d'ailleurs au cour des plus récents modèles conceptuels de la qualité des services dans le domaine de la santé. Il est d'ailleurs connu que les organisations réussissant à établir des relations positives entre gestionnaires et personnel, voire une gestion participative, augmentent la satisfaction au travail et diminuent l'épuisement du personnel ${ }^{35}$. Par ailleurs, selon Mintzberg ${ }^{36}$, les organisations avec une gestion centralisée et rigide - de type mécaniste, comme les CHSLD éprouvent plus de difficultés d'adaptation au changement que celles ayant une gestion décentralisée et flexible.

La démarche participative de prise en compte du collectif de travail dans l'application de programmes innovants est déjà implantée dans d'autres domaines, comme dans celui de la santé au travail. Il est en effet reconnu

32. Marguerite Kelly et Eileen McSweeney, «Re-visioning respite: a culture change initiative in a long-term care setting in Eire», Quality in Ageing, vol. 10, n³, 2009, p. 4-11; Sherry B. Robinson, Richard B. Rosher, «Tangling with the barriers to culture change: creating a resident-centered nursing home environment», Journal of Gerontological Nursing, vol. 32, n 10, 2006, p. 19-25, Jules Rosen, Vikas Mittal, Howard Degenholtz et al., «Ability, incentives, and management feedback: organizational change to reduce pressure ulcers in a nursing home», Journal of the American Medical Directors Association, vol. 7, n³, 2006, p. 141-146.

33. Dale E. Yeatts, Cynthia Cready, Beth Ray, Amy De Witt, et Courtney Queen, «Self-managed work teams in nursing homes: implementing and empowering nurse aide teams», The Gerontologist, vol. 44, n² 2, 2004, p. 256-261.

34. Rosalie A. Kane, «Ethics and the frontline care worker: Mapping the subject», Generations, $n^{\circ} 18,1994$, p. $71-74$.

35. Val Kinjerski, et Berna J. Skrypnek, «The promise of spirit at work: increasing job satisfaction and organizational commitment and reducing turnover and absenteeism in long-term care», Journal of Gerontological Nursing, vol. 34, $\mathrm{n}^{\circ} 10,2008$, p. $17-25$.

36. Henry Mintzberg, Grandeur et décadence de la planification stratégique, Paris, Dunod, 2004. 
depuis vingt ans ${ }^{37}$ que les préposés aux bénéficiaires forment une catégorie professionnelle fortement touchée par des problématiques de santé et sécurité au travail, notamment par un important taux de lésions professionnelles - l'un des plus élevés au Québec ${ }^{38}$. Afin de répondre à ce problème, l'application de formations visant à réduire le taux de lésions doit prendre une dimension partenariale: les préposés, comme tous les autres types de professionnels, doivent faire partie intégrante du processus de diffusion de l'innovation formative ${ }^{39}$, précisément parce que les chances d'appropriation de l'innovation sont plus grandes. Cela est également vrai dans le cadre de l'évaluation des programmes de formation en santé au travail: les acteurs ciblés par la formation devraient en faire partie intégrante ${ }^{40}$. Par ailleurs, Harrisson et Legendre ${ }^{41}$ ont montré que l'innovation en santé et sécurité au travail doit s'encastrer dans la culture organisationnelle, c'est-à-dire en prenant appui sur les procédures, attentes, valeurs et traditions. La compréhension de la culture organisationnelle doit être centrale dans la construction de l'innovation.

En ce sens, il serait important que la pratique partenariale de coconstruction et codiffusion de l'innovation puisse se réaliser non pas seulement en santé au travail, mais aussi dans l'optique des programmes de formation relatifs à la qualité des pratiques. Faire participer les employés, leur donner un pouvoir (empowerment) dans la construction et la diffusion des ces programmes innovants sont donc une force majeure pour une meilleure appropriation des innovations, comme l'ont montré Yeatts et ses collègues ${ }^{42}$.

La cohérence de telles innovations formelles dépend étroitement de leur traduction collective. C'est dire toute l'importance des pratiques des gestionnaires qui détiennent une place majeure pour inciter les acteurs de terrain à s'approprier l'innovation. Il reste que cet idéal nécessite de faire sien deux propositions majeures: d'une part, l'étude sociologique des régulations effectuées par les préposés dans le cadre de leur pratique de travail localisé (dans le service gériatrique, par exemple) est une base compréhensive nécessaire à toute forme d'innovations formelles. D'autre part, il ne faut

37. Marie St-Vincent, Analyse des accidents survenus durant une année dans trois centres hospitaliers, Études et recherche, Montréal, Institut de recherche en santé et sécurité du travail (IRSST), Rapport R-093, 1995, Sylvie Bédard, « Portrait des lésions dans le secteur de la santé et des services sociaux», Objectif Prévention, vol. 35, n 1, 2012, p. 8-9.

38. Marie-Josée Legault et Jacqueline Dionne-Proulx, Problèmes de sécurité au travail, Québec, Télé-Université, 2003.

39. Diane Berthelette, Nicole Leduc, Henriette Bilodeau, Marie-Josée Durand et Cheick Faye, «Evaluation of the implementation fidelity of an ergonomic training program designed to prevent back pain», Applied Ergonomics, vol. 43, n¹, 2012, p. 239-245.

40. Diane Berthelette, Henriette Bilodeau et Nicole Leduc, «Pour améliorer la recherche évaluative en santé au travail», Santé publique, vol. 20, n 3, 2008, p. S171-S179.

41. Denis Harrisson et Camille Legendre, «Introduction», dans Denis Harrisson et Camille Legendre (dir.), Santé, sécurité et transformation du travail, Québec, Presses de l'Université du Québec, 2002, p. 1-8.

42. D. E. Yeatts, C. Cready, B. Ray, A. De Witt et C. Queen, op. cit. 
pas penser l'innovation formative comme l'imposition d'un programme d'un gestionnaire «en haut» envers un travailleur «en bas»; dans une perspective «bottom-up», il faudrait davantage considérer la réalité pratique et informelle du travail des préposés comme la base de construction et de diffusion de ces innovations.

\section{Conclusion}

À partir de l'exemple de la formation AGIR, nous avons montré que les programmes innovants de formation ciblant les préposés aux bénéficiaires individuellement n'occasionnaient pas nécessairement, selon ces derniers, une véritable amélioration des pratiques. Cet échec relatif (car non évalué rigoureusement ici) tient à la non-prise en compte de la dimension collective de la pratique des préposés dans le cadre de la construction et de la diffusion de l'innovation. Nous postulons qu'un tel programme connaitrait de meilleurs résultats si cette dimension collective était prise en considération dans la phase de création et de diffusion de l'innovation. Certains écrits ${ }^{43}$ portant sur l'innovation montrent que ce processus appelle nécessairement une rupture dans les règles, du fait de la nouveauté du produit ou du service demandé, comme les connaissances relatives au maintien de l'autonomie dans le cadre de la formation AGIR. Nous pensons néanmoins que cette rupture doit porter davantage sur le service final à apporter que sur le procédé de construction et de diffusion de l'innovation.

Une étude sociologique des régulations mises en œuvre par un tel groupe d'acteurs professionnels semble être une étape cruciale du processus de construction de programmes innovants. Pour des raisons à la fois politiques et budgétaires, les responsables de ce type de formation ne veulent pas nécessairement se donner les moyens de réaliser ce type d'étude. Par exemple, pour la formation AGIR, le but avoué était de transmettre rapidement aux préposés aux bénéficiaires des compétences jugées manquantes ou oubliées, afin de répondre à une urgence médiatique, celle de la maltraitance des résidants. Il n'était pas question d'implanter ce type de pratiques de manière formelle, par l'intermédiaire du collectif. D'autres programmes, tels que ceux implantés en santé au travail, montrent pourtant que la prise en compte de la participation des préposés à la construction et à la diffusion des innovations est une condition gagnante.

À l'avenir, les études sociologiques portant sur les pratiques des préposés et leur régulation des rythmes de travail pourront adéquatement servir de

43. Constantin Bratianu et Isabelle Bouchardy, «Organisations: entre innovations et normes», Sciences de la société, $n^{\circ} 81,2012$, p. 3-9. 
base empirique à la mise en place de programmes innovants portant sur la qualité des pratiques des préposés, car elles pourront favoriser un meilleur temps d'appropriation. 